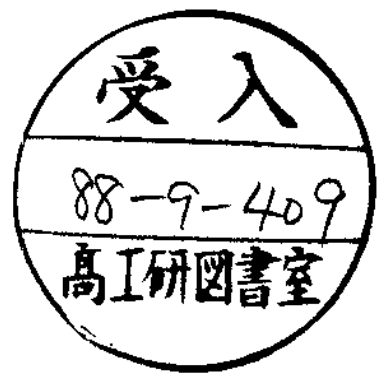

\title{
PRACTICAL LOOK AT NON-COVARIANT GAUGES ${ }^{*}$ )
}

\author{
George Leibbrandt ${ }^{+}$) \\ CERN - Geneva
}

\begin{abstract}
For non-Abelian theories, the dominant non-covariant gauges are the light-cone gauge, the axial gauge and the temporal gauge. These ghost-free gauges are not only useful and viable, but may in certain circumstances even be superior to covariant gauges. In this review, we report on major developments during the past two years. Among the chief issues under discussion today are: (1) the existence of non-local terms and their rôle in an extended BRS formalism; (2) the rôle of ghosts; and (3) a possible unfform prescription for $1 / q \cdot n$ valid for the axial, temporal, planar and light-cone gauges.
\end{abstract}

*) Invited talk presented at the XXIV International Conference on High-Energy Physics, 4-10 August 1988, Munich, West Germany.

+) Permanent address: Department of Mathematics and Statistics, University of Guelph, Guelph, Ontario N1G 2W1, Canada. 


\section{Introduction}

\subsection{Historical Note}

One of the most familiar non-covariant gauges is the Coulomb gauge defined by $\partial_{k} A^{k}(x)=0, k=1,2,3$, with applications mainly in quantum electrodynamics ( $A^{k}$ is the photon field). In non-Abelian models, the dominant non-covariant gauge is the general axial gauge which includes the pure axial gauge, the light-cone gauge and the temporal gauge; the latter are characterized by

$$
\mathrm{n}_{A_{\mu}^{\mu}}^{\mathrm{a}}(\mathrm{x})=0, \mu=0,1,2,3, \mathrm{n}^{2}=\mathrm{n}_{0}^{2}-\overrightarrow{\mathrm{n}}^{2}
$$

where $n_{\mu}=\left(n_{0}, \vec{n}\right)$ is a fixed vector and $A_{\mu}^{a}$ a massless Yang-Mills field, $a=1,2, \ldots, N^{2}-1$ for $S U(N)$. The corresponding gauge-fixing part of the Lagrangian density reads

$$
L_{f i x}=-\frac{1}{2 \alpha}\left(n \cdot A^{a}\right)^{2}, \alpha \rightarrow 0, \alpha \text { gauge parameter } .
$$

The temporal gauge $\left(\mathrm{n}^{2}>0\right)$ had already been used by WEYL [1] in 1929 in the quantization of the Maxwell-Dirac field. In 1949, DIRAC [2] introduced the lightcone gauge $\left(n^{2}=0\right)$ in his paper "Forms of Relativistic Dynamics", which deals with various structures of relativistic dynamical systems. In 1961, KUMMER [3] used for the first time the pure axial gauge $\left(n^{2}<0\right)$ in an article entitled "Gauge Transformation and Quantization of the Free Electromagnetic Field". Between 1960 and 1975, interest in these non-covariant gauges can at best be described as sporadic; they were still being considered "second-class citizens". This unfavourable image was due, in large part, to the unphysical poles of $1 / q \cdot n$ whose proper treatment has challenged the ingenuity and tenacity of theorists ever since.

In the next five years, 1976-1981, there were many more applications in perturbative QCD, also in gravity, but non-covariant gauges remained "under probation", so to speak. The dominant prescription in those days was the principal-value (PV) prescription 


$$
P V \frac{1}{q \cdot n}=\lim _{\varepsilon \rightarrow 0}\left(\frac{1}{q \cdot n+i \varepsilon}+\frac{1}{q \cdot n-i \varepsilon}\right), \varepsilon>0,
$$

which gave reasonable results for the pure axial gauge and the planar gauge $\left(n^{2}<0, n \cdot A^{a}(x)=B^{a}(x)\right)$, but was clearly inappropriate for the light-cone gauge, $\mathrm{n}^{2}=0$. During the past six years, it has become evident that non-covariant gauges are not only useful and viable, but may even be superior, in certain circumstances, to covariant gauges. Non-covariant gauges had finally become "useful citizens".

\subsection{Features}

Non-covariant gauges possess several advantages over their covariant counterparts. For instance, they are ghost-free (hence the occasional terminology "physical gauges"), they can simplify the algebra, specifically in perturbative calculations, and they have succeeded where covariant gauges "failed".

On the other hand, non-covariant gauges are known to require special care; they possess idiosyncrasies otherwise absent in covariant gauges. So the first point is that Feynman integrals are trickier to evaluate. The second point is that the new prescription for the light-cone gauge [4,5] 1mplies the existence of a second light-like vector $n_{\mu}^{*}=\left(n_{0},-\vec{n}\right)$, whose presence "generates" non-local terms.

\subsection{Some Ma jor Issues}

Among the chief issues under discussion today are:

(a) The rôle of the non-local terms alluded to above.

i) Can the BECCHI-ROUET-STORA formalism [6] be modified consistently to incorporate these terms?

ii) What are the implications for the renormalization programme?

(b) What is the rôle of the ghosts? Should one really worry about ghosts, considering that they decouple from physical s-matrix elements?

(c) There is an alarming proliferation of prescriptions for the various gauges, and hence of the data. Is there, perhaps, a unifying prescription which is valid for all four gauges: light-cone, axial, temporal and planar gauge? 


\section{Extended BRS Symmetry for Non-Covariant Gauges}

\subsection{Covariant Gauges}

Extension of the BRS symmetry for covariant gauges was first considered by KLUBERG-STERN and ZUBER [7], and by PIGUET and SIBOLD [8]. These authors enlarged the set of BRS transformations by also transforming the gauge parameter $\alpha$ :

$$
\mathbf{s} \alpha=\chi, \quad \mathbf{s} \chi=0
$$

where $\chi$ is a Grassmann variable, with an assigned Faddeev-Popov charge of +1 , and $s$ is the traditional BRS transformation [9]. The aim was to study, in certain non-Abelian models, gauge dependence and the structure of the counterterms.

\subsection{Non-Covariant Gauges}

a) GAIGG, PIGUET, REBHAN and SCHWEDA [10] examined the extension of the BRS symmetry in the case of non-covariant gauges. They worked with a pure Yang-Mills theory

$$
\begin{aligned}
& \Gamma_{0}=\Gamma_{\mathrm{YM}}+\Gamma_{\mathrm{FP}}, \Gamma_{\mathrm{YM}}=-\frac{1}{4} \int \mathrm{d}^{4} \mathrm{x}\left(\mathrm{F}_{\mu \nu}^{\mathrm{a}}\right)^{2}, \\
& \Gamma_{\mathrm{FP}}=\operatorname{Tr} \int \mathrm{d}^{4} \mathrm{x}\left(\frac{1}{2} \alpha \mathrm{B}^{2}+\mathrm{Bn} \cdot \mathrm{A}-\mathrm{c}_{-} \mathrm{n} \cdot \mathrm{D} \mathrm{c}_{+}\right), \mathrm{D}_{\mu} \mathrm{c}_{+}=\partial_{\mu} c_{+}+i\left[\mathrm{c}_{+}, \mathrm{A}_{\mu}\right], \operatorname{Tr} \equiv \operatorname{trace},
\end{aligned}
$$

where $B$ is a Lagrange multiplier field, $c_{ \pm}$are ghost fields, and $\alpha$ is the gauge parameter. (We shall omit indices whenever their absence does not compromise clarity.) These authors demonstrated that the above theory was invariant under the following extended set of BRS transformations:

$$
\begin{array}{ll}
\mathbf{s A}_{\mu}=\mathrm{D}_{\mu} \mathrm{c}_{+}, & \mathbf{s c _ { + }}=1 \mathrm{c}_{+} \mathrm{c}_{+}, \\
\mathbf{s c _ { - }}=\mathrm{B}, & \mathrm{sB}=0, \\
\mathbf{s} \alpha=\chi, & \mathbf{s} \chi=0, \\
\mathbf{s n}_{\mu}=\phi_{\mu}, & \mathbf{s} \phi_{\mu}=0,
\end{array}
$$

$\chi, \phi_{\mu}$ being Grassmann variables. Equations (2.3a) and (2.3b) correspond to the traditional BRS transformations, (2.3a)-(2.3c) correspond to the enlarged BRS symmetry in the case of covariant gauges, while (2.3a)-(2.3d) express the extended BRS symmetry for axial-type gauges characterized by the non-covariant vector $n_{\mu}$ 
b) Procedure for counterterms. As indicated already, one of the difficulties with physical gauges is the appearance of non-local terms. The latter require the systematic introduction of non-local counterterms, at least in the BRS-context. We shall review the procedure for covariant gauges, because the formalism is more transparent than for non-covariant gauges; besides, there exists a clear account by PIGUET and ROUET [11] which we shall follow here to some extent.

Let $\Gamma$, the generating functional for $1 P i$ vertices, satisfy the Slavnov-Taylor identity

$$
S(\Gamma)=\int d^{4} x\left[\frac{\delta \Gamma}{\delta A_{\mu}} \frac{\delta \Gamma}{\delta J^{\mu}}+\frac{\delta \Gamma}{\delta c_{+}} \frac{\delta \Gamma}{\delta \eta}+\frac{\delta \Gamma}{\delta c_{-}} B\right]=0,
$$

where $J_{\mu}, \eta$ is the source for $s A_{\mu}, s c_{+}$respectively. Next one defines a new quantity $\bar{\Gamma}$,

$$
\Gamma=\bar{\Gamma}-\frac{1}{2} \alpha \int \mathrm{d}^{4} \times \mathrm{B}^{2}
$$

and invokes the quantum action principle [11]. The latter implies the existence of an insertion $\Delta$ :

$$
\begin{aligned}
& \mathrm{S}(\Gamma)=\mathrm{B}_{\bar{\Gamma}}(\vec{\Gamma}) \equiv[\Delta \Gamma]=\Delta+\text { terms of order }(\hbar \Delta), \\
& \mathrm{B}_{\bar{\Gamma}} \equiv \frac{1}{2} \int \mathrm{d}^{4} \mathrm{x}\left[\frac{\delta \bar{\Gamma}}{\delta \mathrm{A}_{\mu}} \frac{\delta}{\delta \mathrm{J}^{\mu}}+\frac{\delta \bar{\Gamma}}{\delta \mathrm{J}^{\mu}} \frac{\delta}{\delta \overline{\mathrm{A}}_{\mu}}+\frac{\delta \bar{\Gamma}}{\delta \mathrm{c}_{+}} \frac{\delta}{\delta \eta}+\frac{\delta \bar{\Gamma}}{\delta \eta} \frac{\delta}{\delta \mathrm{c}_{+}}\right] .
\end{aligned}
$$

The final step is to express the solution of the equation

$$
\mathrm{B}_{\bar{\Gamma}} \Delta=0, \quad\left(\mathrm{~B}_{\bar{\Gamma}_{c \ell}}\right)^{2}=0, \quad c \ell \equiv \text { classical }
$$

in the form

$$
\left.\Delta=\mathrm{B}_{\bar{\Gamma}_{c l}} \mathrm{X}+\mathrm{Y}_{\mathrm{g} . \mathrm{inv}}+\mathrm{O} \mathrm{h} \Delta\right)
$$

here $\vec{\Gamma}_{c l}$ denotes the classical action and $\mathrm{Y}_{\mathrm{g.inv}} \propto \int \mathrm{d}^{4} \mathrm{x}\left(\mathrm{F}_{\mu \nu}^{\mathrm{a}}\right)^{2}, \mathrm{~F}_{\mu \nu}^{\mathrm{a}}$ being the usual field strength tensor. The structure of the functional $X$ is arbitrary, apart from having to possess the right mass dimension and ghost number [12]. If $\Delta$ is given by $(2.8)$, then conventional wisdom asserts that one can determine the counterterms recursively $[11]$. 
The above technique has been applied to superaxial gauges by KUMMER et al. [13] who employ superfields in the context of an $\mathrm{N}=1$ supersymmetric Yang-Mills model. With the help of the quantum action principle they study, among other things, renormalizability and the gauge-independence of the s-matrix.

\section{Non-Local Terms in the Light-Cone Gauge}

\subsection{Some Details}

We demonstrate the procedure of Section $2.2 \mathrm{~b}$ in the case of non-covariant gauges, giving some explicit examples of non-local terms. We shall work in the light-cone gauge $\left(n^{2}=0\right)$, where non-local expressions were first discovered [4]. We recall that the light-cone gauge prescription $[4,5]$

$$
\left.\frac{1}{q \cdot n}\right|^{l c}=\left\{\begin{array}{lll}
\lim _{\varepsilon \rightarrow 0} \frac{1}{q \cdot n+1 \varepsilon s g n} q^{*} n^{*}, & \varepsilon>0, & \text { (Mandelstam) } \\
\lim _{\varepsilon \rightarrow 0} \frac{q \cdot n^{*}}{q \cdot n q^{*} n^{*}+i \varepsilon}, & \varepsilon>0, & \text { (Leibbrandt) }
\end{array}\right.
$$

where $n_{\mu}=\left(n_{0}, \vec{n}\right), n_{\mu}^{*}=\left(n_{0},-\vec{n}\right)$, leads to non-local terms in the divergent parts of the gluon self-energy [4],

$$
\Pi_{\mu \nu}(p)=A\left(p \cdot n^{*} / p \cdot n\right) n_{\mu} n_{\nu}+10 c a 1 \text { terms }
$$

and the three-gluon vertex function [14-16],

$$
\Gamma_{\mu \nu \sigma}(p, 0,-p)=B\left(p \cdot n^{*} / p \cdot n\right) p_{\nu} n_{\mu} n_{\sigma}+C\left(p \cdot n^{*} /(p \cdot n)^{2}\right) n_{\mu} n_{\nu} n_{\sigma}+10 c a 1 \text { terms. }
$$

The coefficients $A, B, C$ depend on the pole term $\Gamma(2-\omega)$ and on various other combinations of $\mathrm{p}^{2}, \mathrm{n} \cdot \mathrm{n}^{*}$, etc.; $\mathrm{p}_{\mu}$ is the external momentum. In order to match the non-local terms in (3.2) and (3.3) by appropriate counterterms, we use (2.8) together with the following ansatz for $x$ [12]:

$$
\begin{aligned}
& \mathrm{x}=\mathrm{x}_{\text {local }}+\mathrm{x}_{\text {non-1ocal }} \text {, } \\
& x_{\text {non-loca1 }}=a_{5}\left[\left(n^{*} \cdot \partial\right)(n \cdot \partial)^{-1} n \cdot \underset{\sim}{A}\right] \cdot \underset{\sim}{J} \cdot n+ \\
& +a_{7} g\left[\left(n^{*} \cdot \partial\right)(n \cdot \partial)^{-1} n_{\tau \sim}^{A^{\tau}}\right] \cdot n^{\lambda} \underset{\sim \lambda}{A} \wedge\left[(n \cdot \partial)^{-1} \underset{\sim \mu}{\vec{J}} n^{\mu}\right], \quad \underset{\sim \mu}{\vec{J}}=\underset{\sim \mu}{J}+n_{\mu} \underset{\sim}{c} \cdot \cdot
\end{aligned}
$$


This ansatz leads to a consistent determination of all divergent constants [12].

\section{$\underline{3.2 \text { Renormalization }}$}

The rôle of non-local terms for the renormalization programme in the light-cone gauge has been investigated by several groups $[17,18]$. For instance, BASSETTO and his collaborators [19], working outside the BRS formalism, find that non-local terms do not contribute to Green functions, an observation also made in Refs. [20] and [21]. BASSETTO et al. conclude, among other things, "... that 1Pi vertices can be made finfte by subtracting a special kind of local and non-local counterterms which will be completely determined."

In summary, one can say that non-local terms are needed to satisfy the Ward identities, and are necessary for a consistent determination of the divergent constants.

\subsection{Related Topics}

(a) In a paper submitted to this conference, BURNEL and VAN DER REST-JASPERS [22] argue that $B R S$ and ant1-BRS symmetries cannot be realized simultaneously in the planar gauge.

(b) ANDRASI and TAYLOR [23], work1ng in the light-cone gauge, obtain a finite answer for the Insertion $\Delta$ in the limit as $\eta \rightarrow 0$, where $\eta$ appears in the expression for $N_{\mu}=n_{\mu}+i \eta\left(n^{*} \cdot \partial\right)^{-1}\left(\nabla^{2}\right)^{-1} \partial_{\mu}$.

(c) A modified prescription for the axial gauge is discussed in a preprint by FAINBERG et al. [24].

\section{General Prescription for the Axial, Planar, Temporal and Light-Cone Gauges}

4.1 The major nuisance with the axial-type gauges is that the prescriptions for the unphysical poles have until now been all different. Use of these different prescriptions has led to duplication of work and frequent confusion in the literature. The existence of separate prescriptions has also complicated comparison, among individual gauges, of such topics as renormalization, BRS invariance and the computation of Feynman integrals. The purpose of this section is to show that the axial-type gauges may be studied in the context of a uniform prescription for $1 / q \cdot n$. To simplify the notation, we shall represent the light-cone, axial and temporal gauges collectively by the letter $\theta, \theta \equiv\{l c, a x, t e m p\}$, and define $n_{\mu}^{\ominus} \equiv n_{\mu}$. This uniform prescription, suggested by the author a few years ago [25], 
reads

$$
\left.\frac{1}{q \cdot n}\right|^{\ominus}=\lim _{\varepsilon \rightarrow 0} \frac{q \cdot n^{\text {dual }}}{q \cdot n q \cdot n^{\text {dual }}+1 \varepsilon}, \varepsilon>0,
$$

where [26]

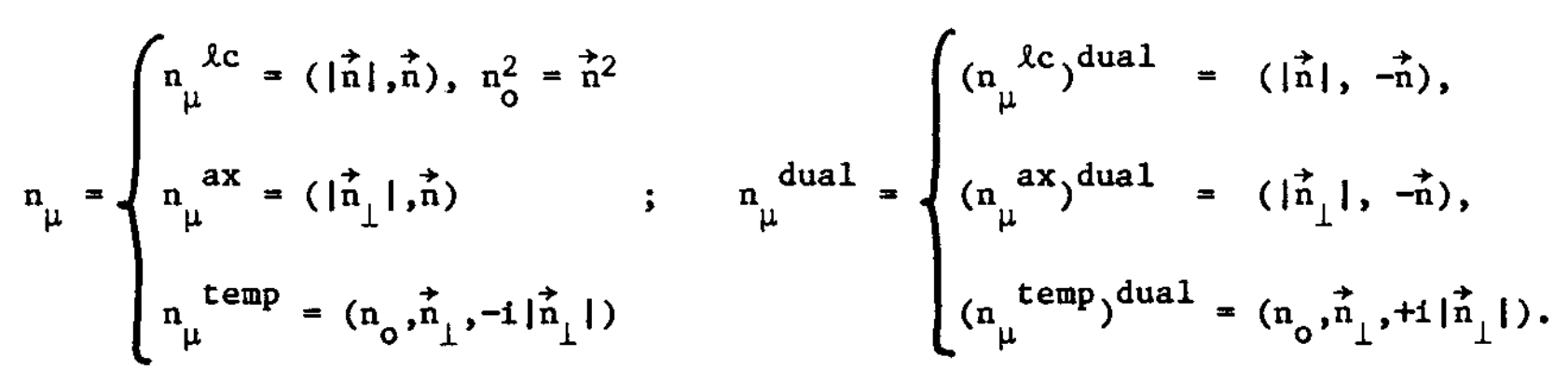

Prescription (4.1) works for double poles [26] and is equally applicable to the planar gauge, a variant of the axial gauge.

Example: To 1llustrate the general structure of the resulting Feynman integrals, consider the integral $\int d^{2 \omega} q q_{\mu}\left[(q-p)^{2} q \cdot n\right]^{-1}, \omega$ complex. Its divergent part (Euclidean space) is given by

$$
\begin{aligned}
& I_{\mu}^{\Theta}=\frac{2 p \cdot F^{\Theta}}{n \cdot F^{\theta}}\left(p_{\mu}-\frac{p \cdot n}{n \cdot F^{\Theta}} F_{\mu}^{\Theta}-\frac{p \cdot F^{\Theta}}{2 n \cdot F^{\Theta}} n_{\mu}+\frac{n^{2} p \cdot F^{\Theta}}{2\left(n \cdot F^{\Theta}\right)^{2}} F_{\mu}^{\Theta}\right) \bar{I}, \\
& \bar{I} \equiv \pi^{2} \Gamma(2-\omega),
\end{aligned}
$$

with $\left(F_{\mu}{ }^{\theta}\right)^{2}=0$. In the temporal gauge, the null vector $F_{\mu}^{\text {temp }}$ reads $F_{\mu}^{\text {temp }}=$ $\left(\vec{n}_{\perp} / \sigma, n_{4} / \sigma, i\right), \sigma=\left(\vec{n}_{\perp}^{2}+n_{4}^{2}\right)^{\frac{1}{2}}$, and in the light-cone gauge, $F_{\mu}^{l c}=n_{\mu}^{*} /|\vec{n}|$. In the latter case, the right-hand side of (4.2) reduces to the "ancient" result [4]

$$
I_{\mu}^{l c}=\frac{2 p \cdot n}{n \cdot n}{ }^{*}\left(p_{\mu}-\frac{p \cdot n}{n \cdot n}{ }^{*} n_{\mu}^{*}-\frac{p \cdot n^{*}}{2 n \cdot n^{*} n_{\mu}}\right) \vec{I} .
$$

\subsection{Applications of the General Prescription}

The unifying prescription (4.1) has been successfully applied to the gluon selfenergy $\Pi_{\mu \nu} \Theta[26]$, the gluon self-energy in the planar gauge $\Pi_{\mu \nu}^{\text {planar }}[27,28]$, and 
to the quark self-energy and quark-quark-gluon vertex [29]. All three processes generate non-local terms. For instance, the planar-gauge gluon self-energy has the structure

$$
\Pi_{\mu \nu}^{\mathrm{Planar}}=\text { local terms }+\frac{\mathrm{p} \cdot \mathrm{F}}{\mathrm{p} \cdot \mathrm{n}}[\cdots]_{\mu \nu}, \mathrm{F}_{\mu}^{\mathrm{ax}} \equiv \mathrm{F}_{\mu} .
$$

To obtain the counterterms in this gauge, we proceed as in Section 3: we write

$$
\Delta=\mathrm{B}_{\bar{\Gamma}_{\mathrm{cl}}}\left(\mathrm{X}_{\mathrm{loc}}+\mathrm{X}_{\mathrm{non}-\mathrm{loc}}\right)+\mathrm{Y}_{\mathrm{g.inv}}+0(\hbar \Delta)
$$

and make the following ansatz for $x_{\text {non-loc }}$ in terms of $n_{\mu}, F_{\mu}$ :

$$
\begin{aligned}
& X_{\text {non-1oc }}=a_{6}\left[(F \cdot \partial)(n \cdot \partial)^{-1} A_{\mu}^{a}\right] \bar{J}^{a}+a_{8}\left[(F \cdot \partial)(n \cdot \partial)^{-1} F \cdot A^{a}\right] \cdot F \cdot \bar{J}^{a}+ \\
& +(3 \text { other non-local terms })+\ldots
\end{aligned}
$$

with $a_{6}=-\left(n^{2} / n \cdot F\right) \lambda, a_{8}=\left(n^{2} /(n \cdot F)^{2}\right) \lambda, \ldots$, and $\lambda=g^{2}(4 \pi)^{-2} C_{Y M} \Gamma(2-\omega) ; \bar{J}$ is the same as in Section 3 . The complete set of divergent constants $\left\{a_{i}\right\}$ is given in Ref. [27].

\subsection{Related Work}

GAIGG, KREUZER, PIGUET and SCHWEDA [30] have likewise generalized the light-cone prescription (3.1). Their approach differs appreciably from ours, so that their momentum integrals look quite different [31]. Nevertheless, the respective answers of the two groups, e.g., for the gluon self-energy $\Pi_{\mu \nu}^{\ominus}$, are very close.

In yet another recent preprint, HÜFFEL, LANDSHOFF and TAYLOR [32] generalize the light-cone prescription (3.1) to the temporal gauge, and then proceed to compute the Wilson loop. They conclude that the time-dependence of the Wilsonloop operator exponentiates to order $g^{4}$, an encouraging result considering that this is a two-loop calculation.

\section{Conclusion}

It is difficult, and would probably be unfalr, to highlight any particular calculation or theoretical analysis in this area during the last few years. There certainly has been a noticeable increase, not only in the number of published papers, but also in their quality and in the range of topics covered. While 
progress since 1982 has been solid, more effort is needed to place the non-covariant gauges on a rigorous mathematical footing.

We have also seen that non-local terms have become a "part of life" and can be handled systematically, at least at the one-loop level. Ghosts continue to lead a dual existence: though decoupling from physical S-matrix elements, they are definitely needed in the BRS formalism. Finally, we have seen that there is a chance of unifying the axial-type gauges by means of a general prescription for $1 / q \cdot n$. If this ambitious programme is to succeed, we will eventually have to verify higher-loop integrals, prove unitarity and renormalizability and convince ourselves that power counting is valid at all orders of perturbation theory.

\section{Acknowledgements}

It gives me great pleasure to thank Professor C. Becchi and Professor M. Schweda for several illuminating discussions. I am also grateful to Professors Maurice Jacob and John Ellis for their kind hospitality during my stay in the Theory Division at CERN. This research was supported in part by the Natural Sciences and Engineering Research Council of Canada under grant No. A8063.

\section{$\underline{\text { References }}$}

1. H. Wey1: Z. Phys. 56, 330 (1929).

2. P.A.M. Dirac: Rev. Mod. Phys. 21, 392 (1949).

3. W. Kummer: Acta Phys. Austriaca 14, 149 (1961).

4. G. Le1bbrandt: Phys. Rev. D29, 1699 (1984).

5. S. Mande1stam: Nuc1. Phys. B213, 149 (1983).

6. C. Becchi, A. Rouet, R. Stora: Phys. Lett. 52B, 344 (1974); Commun. Math. Phys. 42,127 (1975).

7. H. Kluberg-Stern, J.-B. Zuber: Phys. Rev. D12, 467, 482, 3159 (1975).

8. 0 . Piguet, K. Sibold: Nucl. Phys. B248, 301 (1984).

9. C. Itzykson, J.-B. Zuber: Quantum Field Theory (McGraw-Hi11, New York 1980).

10. P. Gaigg, O. Piguet, A. Rebhan, M. Schweda: Phys. Lett. $\underline{B 175}, 53$ (1986).

11. O. Piguet, A. Rouet: Phys. Reports $\underline{76}, 1$ (1981).

12. G. Leibbrandt, S.-L. Nyeo: Nuc1. Phys. B276, 459 (1986).

13. W. Kummer, M. Mistelberger, P. Schaller, M. Schweda: "Renormalization and Gauge-Independence of Superaxial Gauges", Vienna preprint, May 1988. 
14. A. Andraši, G. Leibbrandt, S.-L. Nyeo: Nucl. Phys. B276, 445 (1986).

15. H.C. Lee, M.S. Milgram: Nucl. Phys. B268, 543 (1986).

16. M. Dalbosco: Phys. Lett. B163, 181 (1985).

17. G. Leibbrandt: Rev. Mod. Phys. 59, 1067 (1987).

18. S.-L. Nyeo: Phys. Rev. D34, 3842 (1986); Nuc1. Phys. B273, 195 (1986).

19. A. Bassetto, M. Dalbosco, R. Soldati: Phys. Rev. D36, 3138 (1987).

20. R. Floreanini: Ph.D. Thesis (International School for Advanced Studies, Trieste, Italy, 1985).

21. H.C.Lee, M.S. Milgram: Phys. Rev. Lett. 55, 2122 (1985).

22. A. Burne1, M. Van der Rest-Jaspers: "BRS and Ant1-BRS Symmetries in the Planar Gauge", Liège preprint, May 1988.

23. A. Andraši, J.C. Taylor: Nucl. Phys. $\underline{B 302}, 123$ (1988).

24. V.Ya. Fainberg, S.N. Karnaukhov, E.Kh. Veliev: "Yang-Mills Field Quantization in Modified Axial Gauge", preprint 21, Moscow 1988.

25. G. Leibbrandt: Seminar at the Summer Theoretical Physics Institute in Quantum Field Theory, University of Western Ontario, London, July 28-August 10, 1985.

26. G. Letbbrandt: Nuc1. Phys. B (1988, in press).

27. G. Lelbbrandt, S.-L. Nyeo: Mod. Phys. Lett. A (1988, in press).

28. G. Narde1li, R. Soldati: Phys. Lett. B206, 495 (1988).

29. G. Leibbrandt, S.-L. Nyeo: "Application of Unifying Prescription for AxialType Gauges in QCD", Guelph preprint \#1987-109, December 1987.

30. P. Gaigg, M. Kreuzer, 0. Piguet, M. Schweda: J. Math. Phys. 28, 2781 (1987).

31. P. Gaigg, M. Kreuzer, G. Pollak: "On the Renormalization of Yang-Mills Theories in Axial Gauges within a Uniform Prescription", preprint TU-Wien, April 1988.

32. H. Huffel, P.V. Landshoff, J.C. Taylor: "Wilson-1oop Exponentiation and Temporal Gauges", Vienna preprint \#UWThPh-1988-12 (revised version). 